\title{
iPSC-induced neurons with the V337M MAPT mutation are selectively vulnerable to caspase- mediated cleavage of tau and apoptotic cell death
}

Panos Theofilas ( $\sim$ panos.theofilas@ucsf.edu)

University of California San Francisco https://orcid.org/0000-0001-9701-1352

Chao Wang

Gladstone Institute of Neurological Disease

David Butler

Neural Stem Cell Institute

Dulce 0. Morales

University of California San Francisco

Cathrine Petersen

University of California San Francisco

Andrew J. Ambrose

University of California San Francisco

Brian Chin

Shanghai ChemPartner

Teddy Yang

Shanghai ChemPartner

Shireen Khan

ChemPartner

Raymond Ng

ChemPartner

Rakez Kayed

The University of Texas Medical Branch at Galveston

Celeste M. Karch

Washington University in St Louis

Bruce L. Miller

University of California San Francisco

Jason E. Gestwicki

University of California San Francisco

Li Gan

Weill Cornell Medicine

Sally Temple 
Neural Stem Cell Institute

\section{Michelle R. Arkin}

University of California San Francisco

\section{Lea T. Grinberg}

University of California San Francisco https://orcid.org/0000-0002-6809-0618

\section{Research Article}

Keywords: Tau cleavage, neoepitope antibody, active caspase-6, iPSCs, FTLD, V337M MAPT mutation, tauopathies

Posted Date: April 26th, 2021

DOI: https://doi.org/10.21203/rs.3.rs-300172/v2

License: (c) (i) This work is licensed under a Creative Commons Attribution 4.0 International License. Read Full License 


\section{Abstract}

Background: Tau post-translational modifications (PTMs) are associated with progressive tau accumulation and neuronal loss in tauopathies, including forms of frontotemporal lobar degeneration (FTLD) and Alzheimer's disease (AD). Tau proteolysis by caspases, including caspase-6, represents an understudied PTM that may increase neurotoxicity and tau self-aggregation.

Methods: To elucidate the presence and temporal course of caspase activation, tau cleavage, and neuronal death, we generated two novel epitope (neoepitope) monoclonal antibodies (mAbs) against caspase- 6 tau proteolytic sites. We evaluated tau cleavage and response to apoptotic stress in cortical neurons derived from induced pluripotent stem cells (iPSCs) with frontotemporal dementia (FTD)-causing V337M MAPT mutation. We tested the neuroprotective effect of caspase inhibitors in the induced neurons. We also demonstrated the presence of the tau neoepitopes in postmortem brains from an individual with FTD (V337M MAPT) and an individual with $\mathrm{AD}$, compared to a healthy control.

Results: FTLD V337M MAPT and AD postmortem brains showed positivity for both cleaved tau mAbs and active caspase-6. Relative to isogenic wild-type MAPT controls, V337M MAPT neurons cultured for 3 months showed a time-dependent increase in pathogenic tau in the form of caspase-cleaved tau and phosphorylated ( $p$ )-tau, and higher levels of tau oligomers. Accumulation of toxic tau species in V337M MAPT neurons was correlated with increased vulnerability to pro-apoptotic stress. Notably, this mutationassociated cell death was pharmacologically rescued by inhibition of effector caspases.

Conclusions: Culturing iPSC-derived neurons for three months exposes age-related tau pathologies, including caspase-mediated cleavage, that are also observed in human postmortem brains with abnormal tau deposition. Neoepitope antibodies to caspase-cleaved tau may serve as biomarkers of tau pathology. Furthermore, caspases could be viable therapeutic targets for tau pathogenesis in FTLD and other tauopathies.

\section{Background}

Tau post-translational modifications (PTMs) represent pathological hallmarks of neurodegenerative diseases collectively known as tauopathies, including frontotemporal lobar degeneration (FTLD) and Alzheimer's disease (AD) [1, 2]. Considering the absence of effective treatments against tauopathies, therapeutic interventions that delay or impede tau pathogenesis are an urgent medical need. Tau hyperphosphorylation (p-tau) remains one of few PTMs extensively investigated in tauopathies [3, 4], despite evidence of significant involvement of other tau PTMs, such as those generated by proteolytic cleavage of tau by active caspases (cysteine-aspartic proteases). In experimental models, caspasecleaved tau promotes tau aggregation, tau spreading, and neurotoxicity [5-8].

Caspases are proteolytic enzymes with well-defined roles in cell death and inflammation. Apoptotic caspases are broadly classified as either initiator caspases that activate their effector counterparts or effector/executioner caspases that induce cell death [9-11]. Accumulating evidence support a key 
pathogenic role of the effector caspase- 6 in major diseases including AD, Huntington disease, and ischemic stroke [12-15]. In AD, active caspase- 6 is associated with three stages of cellular dysfunction, including inflammation [16], apoptosis, and tau cleavage $[8,17,18]$. We previously demonstrated a positive linear correlation between AD neuropathological stages and increasing levels of active caspase- 6 and $p$-tau colocalization in the locus coeruleus and dorsal raphe nucleus, brain regions showing the earliest vulnerability to AD-tau pathology [15]. Moreover, active caspase- 6 was found to colocalize with NFT-positive neurons in $A D$ patients' brains $[15,17,19,20]$, promote axonal degeneration in several cellular and in vivo animal models of $A D[21,22]$, and its levels inversely correlate with cognitive performance in aged individuals [23]. While other caspases are likely also involved in neurodegenerative disease, caspase- 6 inhibition may be sufficient to reduce neuronal damage with a significant therapeutic index, since caspase- 6 knockout animals are healthy and protected from AD pathology or proinflammatory stimuli $[16,24]$.

Active caspase- 6 may promote NFT formation by cleaving tau after aspartic acid (D) at multiple sites, including D421 near the C-terminus $[8,17,25]$. Tau cleaved at D421, which can be targeted by several caspases, has a stronger seeding propensity than full-length tau and is a component of the core of paired helical filaments (PHFs) in AD [5, 26, 27]. Caspase-6 cleaves tau at additional sites, including D13 [18] and D402 [23, 28, 29], which are also found to mediate tau aggregation and toxicity in vitro. Yet, these sites are underexplored compared to D421, mainly due to a lack of available monoclonal antibodies (mAbs). Currently, D13 is detected by the loss of $\mathrm{N}$-terminal tau epitopes recognized by the antibodies Tau-12 and 5A6 [18], and D402 is detected using a polyclonal antibody [29].

To elucidate the role of caspase activation, caspase- 6 tau cleavage, and neuronal death in tauopathies, we generated induced cortical neurons (iNs) from induced pluripotent stem cells (iPSCs) with the FTDcausing V337M MAPT mutation (tau ${ }^{\mathrm{V} 337 \mathrm{M}}$ ) [30-33] and WT isogenic controls (tau ${ }^{\mathrm{WT}}$ ). iPSCs and their subsequent conversion to iNs by expression of neuronal transcription factors offer a clinically-relevant model of human tau pathogenesis in a disease-specific genetic background [32, 34-37]. We evaluated tau proteolysis using newly developed novel epitope (neoepitope) mAbs against caspase-cleaved tau at D13 and D402 sites in the iNs and human postmortem brains with tau accumulation. Our results demonstrate neoepitope mAb positivity in human brains from individuals with AD and FTLD-tau, a timedependent accumulation of caspase-cleaved tau, and increased vulnerability to acute apoptotic stress in

the tau ${ }^{\mathrm{V} 337 \mathrm{M}}$ iNs that is reversed by inhibition of effector caspases, including caspase- 6 . This study offers insights into the potential of caspase- 6 and other caspases as targets for therapeutic intervention against tau pathology in FTLD and other tauopathies.

\section{Methods}

\section{Development of caspase- 6 cleaved tau neoepitope monoclonal antibodies}


To address the absence of mAbs against tau sites primarily cleaved by caspase- 6 , we generated neoepitope mAbs against cleaved tau at D402 (mAbD402; 1-402) and D13 (mAbD13; 14-441) (Fig. 1a). We generated the mAbs by immunizing 6-8-week-old wild type Balb/c and SJL mice (SLAC) with keyhole limpet hemocyanin $(\mathrm{KLH})$ conjugated tau peptides using protocols approved by the ChemPartner IACUC committee. $50 \mu \mathrm{g}$ of each peptide was injected into the abdominal cavity of each mouse along with 0.25 $\mathrm{mL}$ Complete Freund's Adjuvant (Sigma). To enhance the immune response, $25 \mu \mathrm{g}$ of KLH conjugated tau peptides were injected into the abdominal cavity of each mouse along with $0.25 \mathrm{~mL}$ Incomplete Freund's Adjuvant (IFA) two weeks after the first immunization, and subsequent boosts were administered three weeks apart. Blood samples from each mouse were collected one week after each immunization. The antibody titer and specificity were determined by enzyme-linked immunosorbent assay (ELISA) analysis and immunoblotting (see Supplemental Experimental Procedures).

Mice with specific immune response against tau peptides and proteins were selected for fusion and were given a final boost by intraperitoneal injection of $100 \mu \mathrm{g}$ of the corresponding immunogen. After four days, mice were sacrificed and single-cell suspensions of splenocytes were prepared in $\mathrm{NH}_{4} \mathrm{OH}$ at $1 \%$ $(\mathrm{w} / \mathrm{w})$, followed by centrifugation at $1000 \mathrm{rpm}$ and washes with DMEM (Invitrogen). Viable splenocytes were fused with mouse myeloma cells SP2/0 (ATCC) at a ratio of 5:1 with high-efficiency electric fusion (BTX ECM200). Fused cells were re-suspended in DMEM with 20\% FBS and hypoxanthine-aminopterinthymidine (HAT) medium (Invitrogen). 14 days after cell fusion, hybridoma supernatants were collected and screened by ELISA. Clones with an OD450 nm >1.0 were expanded in a 24-well plate containing DMEM with $10 \%$ heat-inactivated FBS, and supernatants were collected after 3 days of culture. The antibody isotypes were determined, and ELISA and western blot were used to test their ability to bind to tau. Clones that showed desired reactivity and specificity against tau were subjected to subcloning to produce stable monoclonal hybridoma cells. Sub-cloning was carried out by limited dilution in a 96-well plate with DMEM media containing 10\% FBS. Clones with specific Tau binding were further expanded in DMEM media containing 10\% FBS for subsequent antibody production with Protein A purification.

Hybridoma cells were cryopreserved for future production. Reactivity and specificity of purified mAb.D13 and mAb.D402 were confirmed by ELISA against tau proteins and peptides (Fig. S1) and western blot analysis against recombinant tau proteins (Fig. S2-a, b).

\section{Human postmortem tissue processing and immunohistochemistry}

Paraffin-embedded tissue sections cut at $8 \mu \mathrm{m}$ from the temporal cortex of an individual with tau ${ }^{\mathrm{V} 337 \mathrm{M}}$ and FTD (68 years, female), a patient with sporadic late-stage AD and severe dementia (64 years, female, $A D$ neuropathologic change: $A 3 B 3 C 3$ ), and a healthy control free of neuropathological diagnosis (82 years, female, AD neuropathologic change: A1B1C0), were sourced from the Neurodegenerative Disease Brain Bank at the University of California, San Francisco. Immunohistochemistry was performed on deparaffinized and rehydrated sections, following quenching of endogenous peroxidases with $3 \% \mathrm{H}_{2} \mathrm{O}_{2}$ in methanol (Sigma) for 30 min and antigen retrieval in Tris-EDTA buffer/PBS with 0.05\% Tween (Sigma) 
for $5 \mathrm{~min}$ at $121 \mathrm{C}$ in the autoclave. Sections were blocked in 5\% milk/PBS with $0.05 \%$ Tween for 30 min and incubated with primary antibodies (TauC3 (D421), Invitrogen, AHB0061, mouse, 1:500; Active caspase-6 (cleaved at Asp179), Aviva Systems, OAAF05316, rabbit, 1:500; mAb.D402, ChemPartner, clone 47G7B5, mouse, 1:500; mAb.D13, ChemPartner, clone 5G4-1C5, mouse, 1:500) overnight at room temperature. After three washes with PBS-T (0.05\% Tween-20), sections were incubated with the corresponding biotinylated secondary antibodies, followed by DAB or Red AP chromogen incubation based on manufacturer's instructions (Vector labs) and counterstained with hematoxylin (Sigma).

\section{Cell lines}

iPSC-derived induced neurons (iNs) with heterozygous V337M MAPT mutation (tau ${ }^{\mathrm{V} 337 \mathrm{M}}$ ) and WT isogenic controls $\left(\operatorname{tau}^{\mathrm{WT}}\right)$ were generated as previously described $[32,38]$. The Neurogenin 2 (Ngn2)integrated iPSC line was created from a tau ${ }^{\mathrm{WT}}$ human iPSC line (male; WTC11) [38, 39]. The Tet-ON 3Gcontrolled Ngn2 transgene was integrated into the AAVS1 locus of human iPSC lines through a TALEN nuclease pair [38]. CRISPR/Cas9 gene editing by homologous recombination was used to introduce the tau ${ }^{\mathrm{V} 337 \mathrm{M}}$ into the Ngn2-integrated iPSCs. Briefly, iPSCs were transfected with the Human Stem Cell Nucleofector Kit (Lonza) with sgRNA (5'-CTTGTGGGGTCA-TGGTTTACAGG-3') plasmid (Addgene, 68463), Cas9 plasmid, and donor DNA plasmid containing a neomycin-resistance cassette (adapted from Addgene, PL552). Transfected cells were selected with neomycin for one week. Neomycin-resistant clones were selected and verified by genomic PCR and DNA sequencing. After sequence validation of the

tau $^{\mathrm{V} 337 \mathrm{M}}$ site, $1 \mathrm{mM}$ Cre recombinase (Excellgen) was added to remove the neomycin-resistance cassette from gene-targeted iPSCs. Moreover, the top 10 potential off-target sites were sequenced and for each genotype, the correct clones were karyotyped (Cell Line Genetics) and expanded for subsequent experiments. iNs were differentiated using a two-step protocol as previously described [32, 38]. See Supplemental Experimental Procedures for detailed methods.

\section{Western blot protein analysis}

Western blot analysis was performed using the Mini-PROTEAN Tetra system (Biorad) and standard immunoblotting techniques. See Supplemental Experimental Procedures for detailed methods.

\section{Cell assays and compound treatment}

iNs cultured in 96-well plates were treated with either vehicle (DMSO) or staurosporine (STS) and the pancaspase inhibitor z-VAD-fmk (Enzo Lifesciences) directly in the media. Following $6 \mathrm{~h}$ treatment, caspase3/7 levels were examined using Caspase-Glo 3/7 (Promega) according to the manufacturer's instructions.

An ELISA based on lamin A cleavage, a specific substrate of active caspase-6, was used for detection and quantification of caspase-6 activity as previously described [40]. Briefly, iNs were treated with either vehicle (DMSO) or staurosporine (STS) and the caspase-6 inhibitor z-VEID-fmk (Enzo Lifesciences) directly in the media for $48 \mathrm{~h}$. Next, cells were fixed with $4 \%$ paraformaldehyde (Thermo Fisher Scientific) in PBS for 15 min and blocked with 5\% bovine serum albumin (Sigma) in PBS with $0.01 \%$ Triton X-100 for $1 \mathrm{~h}$ at room temperature. Cells were then incubated with cleaved lamin A primary antibody (Cell Signaling, 
2035, rabbit, 1:200) overnight at $4^{\circ} \mathrm{C}$, followed by an HRP-conjugated secondary antibody incubation (Invitrogen) for $1 \mathrm{~h}$ at room temperature (GE Healthcare, NA934V, donkey anti-rabbit, 1:1000) a 5 min incubation with chemiluminescent HRP substrate (Thermo Fisher Scientific), and reading with a Spectramax microplate reader (Molecular Devices). iN cytotoxicity was measured using lactate dehydrogenase (LDH) release assay (Promega) following 48h treatment with vehicle (DMSO), STS, or STS/ z-VAD-fmk according to the manufacturer's instructions. Readings were performed with a Spectramax microplate reader, followed by western blot or immunocytochemistry analysis.

To examine the morphological effects of STS and the z-VAD-fmk treatment in the iNs, we performed automated quantification of neurite length using IN Cell Developer Toolbox analysis routines based on image stacks uploaded from the IN Cell Analyzer 6500 confocal imager (GE Healthcare). To accommodate the treatment-induced variation of neurite morphology during image acquisition, the Software Autofocus function was chosen to optimize the focal plane for each imaging field. DAPI was used for nuclei detection and microtubule-associated protein 2 (MAP2) for the detection of the cytoplasm and neurites. Mean neurite length per cell was calculated by subtracting a binary image of the cell body from a binary image of the entire cell, including the neurites. Data were obtained from 3-7 replicate wells per condition and an average of $1.5-2 \mathrm{~K}$ cells per well. For IF analysis, fixed neurons were imaged using an IN Cell Analyzer $6500 \mathrm{HS}$ confocal imager (GE Healthcare). For further details see Supplemental Experimental Procedures.

\section{Statistics}

Statistical analyses and graphics were generated using R Statistical Software (version 3.6.1; R Foundation for Statistical Computing, Vienna, Austria). Groupwise differences were analyzed by comparing means (student's t-test, one or two-way ANOVA with post hoc Tukey tests) with the family-wise error rate set to 0.05 to correct for multiple comparisons. Data from at least three independent experiments are presented as mean \pm SEM.

\section{Results}

\section{Generation of caspase- 6 cleaved tau neoepitope monoclonal antibodies}

Besides TauC3 (D421), mAbs that recognize specific caspase-cleaved tau epitopes are crucially missing. Here, we developed two neoepitope mAbs against D13 and D402 tau cleavage sites (Fig. 1a) using hybridoma technology. Neoepitope antibodies selectively bound to cleaved tau (1-402 or 14-441) but not full-length tau (1-441) as detected by ELISA and western blot analyses (Fig. S1; S2-a, b; See Supplemental Experimental Procedures). Antibody specificity was also confirmed by immunofluorescence (IF) and antigen competition assays where mAb.D402 and mAb.D13 primary antibodies were preincubated with the cognate peptides used as immunogens (Table S1), resulting in loss of antibody signal (Fig. S2-c). Together with TauC3, mAb.D402, and mAb.D13 neoepitope antibodies were used to identify caspasemediated pathological changes in postmortem brains and in tau ${ }^{\mathrm{V} 337 \mathrm{M}}$ and tau ${ }^{\mathrm{WT}}$ iNs. 


\section{Tau pathological changes in postmortem brains of FTLD and AD patients}

We explored whether tau pathological changes are present in human postmortem brains with tau accumulation, including a case with the same tau ${ }^{\mathrm{V} 337 \mathrm{M}}$ mutation as in the iN model described below. We immunostained sections from the temporal cortex of an FTLD tau ${ }^{\mathrm{V} 337 \mathrm{M}}$ carrier and an AD patient, both at end-stage disease, and a healthy elderly control using multiplex immunohistochemistry targeting active caspase- 6 and caspase-cleaved tau markers (Fig. 1b). We detected active caspase- 6 positivity in the cytoplasm and neurites in both disease cases (brown), but not in the healthy control even after prolonged incubation with the chromogen to confirm the absence of signal (Fig. 1b vii-ix). The FTLD tau ${ }^{\mathrm{V} 337 \mathrm{M}}$ carrier was also positive for TauC3 and mAb.D402 (red) in the cytoplasm and neurites that partially co-occurred with active caspase-6. AD brain sections showed strong positivity for all caspase-cleaved tau markers, including mAb.D13 that was absent in FTLD. Such variation on tau pathological burden is expected among individuals with different tauopathies. Moreover, neurons in the AD sections showed partial overlap between cleaved-tau and active caspase- 6 in cytoplasm and neurites. Taken together, our results show that neoepitope mAbs label pathological tau inclusions in AD and FTLD-tau and corroborate the presence of active caspase- 6 in the human brain seen in earlier studies [15].

\section{Characterization of iPSC-induced neurons}

Considering that tau ${ }^{\mathrm{V} 337 \mathrm{M}}$ causes FTD in humans, we generated heterozygous tau ${ }^{\mathrm{V} 337 \mathrm{M}}$ iNs and isogenic tau $^{\mathrm{WT}}$ controls to examine the disease mechanisms involving caspase activation and cleaved tau pathology in a clinically relevant cell culture model. Neuronal induction of a well-characterized human tau $^{\text {WT }}$ line was performed using TALEN-based integration of a doxycycline-inducible Ngn2 transgene into the AAVS1 safe harbor as previously described [32, 38]. CRISPR/Cas9 genome editing was used to introduce the tau ${ }^{\mathrm{V} 337 \mathrm{M}}$ into tau ${ }^{\mathrm{WT}}$ iPSCs to study the mutation's effects in isolation from the donor's genetic background. Both iPSC groups had a normal karyotype (Fig. S3-a) and typical colony-type morphology (Fig. 2a). Moreover, genomic DNA sequencing of tau ${ }^{\mathrm{V} 337 \mathrm{M}}$ and tau ${ }^{\mathrm{WT}}$ iPSCs confirmed the presence of the heterozygous tau ${ }^{\mathrm{V} 337 \mathrm{M}}$ in exon 12 (Fig. S3-b), and homogeneous expression of the pluripotency markers NANOG, OCT4, and SOX2 (Fig. S3-C) in the iNs confirmed the absence of NGN2 expression leakage without the addition of doxycycline. Following doxycycline treatment, iNs exhibited neuron-like morphology in 5-7 days and mature neuronal morphology between 3-4 weeks (Fig. 2a).

One month-old iNs were positive for the microtubule-associated protein 2 (MAP2) neuronal marker in the cytoplasm and neurites, and for the deep and upper cortical nuclear markers CTIP2 and SATB2, respectively, as confirmed by IF (Fig. 2b). iNs were also positive for the glutamatergic marker vesicular glutamate transporter 1 (VGlut1) in the cytoplasm and neurites, an anticipated outcome of the NGN2 expression [32, 41]. Overall, our findings demonstrate that at one-month post-differentiation, iNs exhibit neuron-specific morphology and express cortical and glutamatergic markers.

Increased levels of pathological tau in the tau ${ }^{\mathrm{V} 337 \mathrm{M}}$ neurons 
Since FTLD-tau is characterized by progressive accumulation of toxic tau species and neuronal loss, we examined the presence and temporal course of tau pathological accumulation of the tau ${ }^{\mathrm{V} 337 \mathrm{M}}$ in the mutant iNs relative to WT isogenic controls. We compared iNs cultured from 1 to 3 months using western blot and a panel of tau antibodies, including total and oligomeric tau, 3R and 4R tau, and caspasecleaved tau (Fig. 3).

Protein analysis using the total tau antibody HT7 showed positivity for distinct molecular weight bands corresponding to separate tau isoforms (Fig. 3a). Tau antibody specificity was confirmed by the recombinant human tau ladder included here as an approximate guide of the tau isoform placement as it lacks the tau PTMs present in the cell lysates that could influence tau molecular weight. In agreement with previous studies showing enriched $3 R$ tau levels in early neuronal development [42, 43], we detected predominantly $3 \mathrm{R}$ isoform expression in the iNs and minimal $4 \mathrm{R}$ tau levels, both in tau ${ }^{\mathrm{V} 337 \mathrm{M}}$ and tau ${ }^{\mathrm{WT}}$ iNs (Fig. 3a). Antibody specificity for 3R and 4R tau was confirmed by the positivity of the respective isoform bands in the recombinant tau ladder. Total tau levels were comparable between the mutant and control iNs (Fig. S4a), suggesting that any changes in the amount of pathological tau between the two groups are likely MAPT mutation-dependent and not due to changes in the overall tau levels.

Oligomeric tau aggregates could represent highly toxic and pathologically significant tau species in tauopathies [44]. We therefore investigated the presence and temporal changes of oligomeric and misfolded tau aggregates recognized by the antibody T18 [45] and non-denaturing conditions to preserve the original folded state of tau (Fig. 3b). To estimate the molecular weight of the native proteins, we used a protein standard for native electrophoresis stained with Coomassie Blue for band visualization. We detected elevated oligomeric tau levels in tau ${ }^{\mathrm{V} 337 \mathrm{M}}$ iNs relative to controls at 2 and 3 months post differentiation. These results were reproduced in three independent experiments (Fig. S4-b-d). The protein standard revealed bands at 480-1,048 kD molecular weight, corresponding to tau oligomers, with no tau detected below that range. Conformational tau species identified by the MC1 antibody [46] represent one of the most commonly detectable pathological features of tauopathies. Using the same experimental approach, we probed iN lysates using native electrophoresis. We observed a similar band pattern to T18, with elevated levels of conformational tau present after two months of culture only in the mutant iNs (Fig. 3b, S4 b-d). Overall, our results indicate a time-dependent increase of tau toxic species in the form of oligomeric and conformationally-modified tau in the mutant iNs compared to controls at two months post differentiation, but not earlier.

Next, we examined the temporal changes in p-tau and caspase-cleaved tau levels in the iNs using the anti-phospho-tau mAb PHF1 (Ser396/404), an epitope that is phosphorylated early in tau inclusion formation in humans [47], and three caspase-cleaved tau mAbs, including cleavage sites primarily targeted by caspase-6, D402 and D13, and the D421 site cleaved by multiple caspases $[8,17]$. Semiquantitative analyses of GAPDH-normalized band intensities revealed a 2.5-fold increase of PHF-1 levels in tau ${ }^{\mathrm{V} 337 \mathrm{M}}$ relative to controls at three months post differentiation but not earlier (Fig. 3c). Similarly, caspase-cleaved tau levels showed a 2.5 to 3-fold increase of D421 and D402-positive bands and a 2-fold 
increase of the D13 in tau ${ }^{\mathrm{V} 337 \mathrm{M}}$ iNs relative to controls at three months post differentiation. Again, these differences were absent in younger cells (Fig. 3d-f). Altogether, our results demonstrate a significantly higher accumulation of $p$-tau and caspase-cleaved tau in tau ${ }^{\mathrm{V} 337 \mathrm{M}}$ iNs relative to controls that accumulated at three months post differentiation, but not at earlier stages.

\section{Caspase inhibition is neuroprotective against stress-induced cytotoxicity in the tau ${ }^{\mathrm{V} 337 \mathrm{M}}$ neurons}

To test whether tau ${ }^{\mathrm{V} 337 \mathrm{M}}$ iNs are more vulnerable to stressors relative to controls, we treated three-month tau $^{\mathrm{V} 337 \mathrm{M}}$ iNs with increasing concentrations of the wide-spectrum kinase inhibitor and apoptosis-inducer staurosporine (STS) for up to $48 \mathrm{~h}$. Treatment was followed by detection of cytotoxicity levels measured by lactate dehydrogenase (LDH) release (Fig. S5). We observed a 2-fold increase in cytotoxicity levels in the tau ${ }^{\mathrm{V} 337 \mathrm{M}}$ iNs relative to tau ${ }^{\mathrm{WT}}$ controls, using $40 \mu \mathrm{M}$ STS for $48 \mathrm{~h}$ (Fig. S5-b), and selected this condition for further studies. Cytotoxicity was significantly reduced by treatment with the pan-caspase inhibitor z-VAD-fmk ( $300 \mu \mathrm{M}$ for $48 \mathrm{~h}$ or $4 \times 75 \mu \mathrm{M}$ in $12 \mathrm{~h}$ intervals; Fig. S5-c), indicating that cell death occurred via apoptosis.

Based on these established conditions (Fig. S5), we exposed iNs to $40 \mu \mathrm{M} \mathrm{STS}$ for $48 \mathrm{~h}$ and increasing doses of Z-VAD-fmk (300 and $600 \mu \mathrm{M}$; Fig. 4). Tau ${ }^{\mathrm{V} 337 \mathrm{M}}$ and tau ${ }^{\mathrm{WT}} \mathrm{iNs}$ treated with vehicle (DMSO) or zVAD-fmk $(600 \mu \mathrm{M})$ in the absence of STS showed low LDH release (Fig. 4); hence, baseline levels of apoptosis were low for iNs cultured for three months. Following STS treatment, however, we observed almost a 5 -fold increase in cytotoxicity in the tau ${ }^{\mathrm{V} 337 \mathrm{M}}$ iNs compared to a 4 -fold increase in control iNs. The mutant group was significantly more vulnerable to cytotoxicity following acute stress by STS $(\mathrm{p}<$ 0.001). Moreover, STS co-treatment with z-VAD-fmk significantly reversed cytotoxicity levels in the mutant iNs. Overall, our results demonstrated increased vulnerability to apoptotic cell death in tau ${ }^{\mathrm{V} 337 \mathrm{M}}$ iNs that was ameliorated by caspase inhibition.

To assess specific changes in the activity of caspase -6 and $-3 / 7$ between the tau ${ }^{\mathrm{V} 337 \mathrm{M}}$ and tau ${ }^{\mathrm{WT}}$ iNs following stress exposure, we treated three-month tau ${ }^{\mathrm{V} 337 \mathrm{M}}$ and control iNs with STS and measured caspase activity. Caspase- 6 activity was measured by ELISA for cleaved lamin A, a selective substrate of caspase- 6 over other caspases [48], while Caspases-3/7 activity was measured using a DEVDaminoluciferin substrate assay (Fig. 4). We observed a 4-fold increase in caspase-6 activity in the tau ${ }^{\mathrm{V} 337 \mathrm{M}}$ iNs compared to a 1 -fold increase in control iNs following $20 \mu \mathrm{M}$ STS treatment for $48 \mathrm{~h}$ (Fig. 5a), indicating that the mutant group had significantly higher caspase-6 activity relative to controls $(p<0.01)$. Notably, STS co-treatment with $10 \mu \mathrm{M}$ of the caspase- 6 inhibitor z-VEID-fmk significantly reversed caspase- 6 activity levels in the mutant iNs ( $p<0.001, z$-VEID-FMK/STS vs STS alone). We also observed a 6 -fold increase in active caspase-3/7 levels (Fig. 5b) in the tau ${ }^{\mathrm{V} 337 \mathrm{M}}$ and tau ${ }^{\mathrm{WT}}$ treated with 40 $\mu \mathrm{M}$ STS for $6 \mathrm{~h}$; there was no significant difference between caspase-3/7 activity between $\operatorname{tau}^{\mathrm{V} 337 \mathrm{M}}$ and tau $^{\mathrm{WT}} \mathrm{iNs}$. Caspase activity was reversed to baseline levels after the addition of $300 \mu \mathrm{M}$ of the pancaspase inhibitor Z-VAD-fmk. Overall, these results demonstrate that STS treatment induced apoptotic cell 
death and a marked increase in caspase activity in the iNs that was suppressed by caspase inhibition. Caspase-6, but not caspases-3/-7, was preferentially activated in the tau ${ }^{\mathrm{V} 337 \mathrm{M}}$ iNs relative to controls.

Next, we performed western blot analyses using lysates of iNs treated with $40 \mu \mathrm{M}$ STS and $300 \mu \mathrm{M} \mathrm{z-}$ VAD-fmk (Fig. 5c-d). Following STS treatment, we observed a statistically significant 2-fold increase in

TauC3 binding in the tau ${ }^{\mathrm{V} 337 \mathrm{M}}$ iNs treated with STS compared to vehicle-treated cells $(p<0.001)$. TauC3 binding was not increased in control iNs treated with STS. In line with the cytotoxicity data (Fig. 4), STS co-treatment with z-VAD-fmk in tau ${ }^{\mathrm{V} 337 \mathrm{M}} \mathrm{iNs}$ reversed TauC3 binding to baseline levels.

\section{Stress-induced reduction of neurite length is rescued by caspase inhibition}

The reduction in neurite length is a morphological indicator of compromised cell viability and neurotoxicity $[34,49]$. To examine the phenotypic effects of STS and z-VAD-fmk treatment on neurites in mutant and control iNs, we used immunocytochemistry (ICC) with DAPI to label cell nuclei (blue), and MAP2 (red) to label cytoplasm and neurites (Fig. 6a). Cells were imaged and subjected to automated quantification of neurite length under the same treatment conditions as our cytotoxicity assay (Fig. 4). We observed comparable mean neurite length in untreated iNs; neurite lengths were reduced upon treatment with $40 \mu \mathrm{M}$ STS by 1.6 -fold for tau ${ }^{\mathrm{V} 337 \mathrm{M}}(\mathrm{p}<0.0001)$ and 1.3-fold $(p<0.0001)$ for tau ${ }^{\mathrm{WT}}$ (Fig. 6b). Co-treatment of STS with z-VAD-fmk partially restored neurite length and preserved MAP2positive processes in tau ${ }^{\mathrm{V} 337 \mathrm{M}}$ iNs compared to STS-treated cells $(p<0.01)$. For tau ${ }^{\mathrm{WT}}$ iNs, neurite length in STS treated cells was also restored upon the addition of Z-VAD-fmk $(p<0.05)$. These data further demonstrate that neurotoxicity following STS treatment in the iNs was significantly caspase-dependent.

\section{Discussion}

Active caspases can directly cleave tau, generating tau species with an enhanced propensity for selfaggregation and accumulation into neuronal cytoplasmic inclusions $[8,18,29,50]$. Most of this evidence comes from studies using animal models and immortalized cell lines that do not necessarily recapitulate the clinical phenotype. Our study innovates by using (a) a disease-relevant, iPSC-derived human neuronal model with the FTLD-causing tau ${ }^{\mathrm{V} 337 \mathrm{M}}$, (b) neoepitope mAbs to detect caspase- 6 cleaved tau species, and (c) pharmacological intervention against caspase activity. Different from most studies, we maintained tau ${ }^{\mathrm{V} 337 \mathrm{M}}$ and tau ${ }^{\mathrm{WT}}$ iNs for up to three months to avoid capturing artefactual effects caused by age-dependent increases in expression of $p$-tau in developing neurons $[42,51]$. We additionally see an age-dependent accumulation of pathogenic tau, including cleaved tau, $\mathrm{p}$-tau, and oligomeric tau, in cells containing pathogenic tau ${ }^{\mathrm{V} 337 \mathrm{M}}$. Of particular interest, three-month tau ${ }^{\mathrm{V} 337 \mathrm{M}}$ iNs showed a three-fold increase in caspase-cleaved D402 and D13 tau protein levels and increased p-tau and oligomeric tau species compared to controls. These mutant cells were also more sensitive to caspase- 6 activation and apoptosis following acute treatment with STS. We furthermore confirmed the presence of active caspase6, and D13 and D402 tau cleavages in postmortem tissue from patients with AD and MAPT V337M FTLD-tau. In contrast to the high levels of caspase- 6 seen only in the tau ${ }^{\mathrm{V} 337 \mathrm{M}}$, activation of caspase- 3 
and -7 was seen in both tau ${ }^{\mathrm{V} 337 \mathrm{M}}$ and tau ${ }^{\mathrm{WT}}$ iNs after treatment with STS. The selective upregulation of active caspase- 6 suggests that increased cell death following acute stress in the tau ${ }^{\mathrm{V} 337 \mathrm{M}}$ iNs could be caspase- 6 related. For example, previous studies in human primary neurons showed that caspase- 6 but not caspase- 3 is activated by serum deprivation and induces cell death following recombinant active caspase- 6 and -3 microinjection $[52,53]$.

Our data are in alignment with earlier studies using iNs with MAPT mutations that show increased vulnerability to stressors in mature iNs cultured for at least two months [37]. iNs with tau ${ }^{\mathrm{V} 337 \mathrm{M}} \mathrm{MAPT}$ mutation are selectively vulnerable to stressors, likely due to increased pathogenic tau levels and a lower capacity to survive additional stress relative to WT controls [34]. Tau ${ }^{\mathrm{V} 337 \mathrm{M}} \mathrm{iNs}$ also showed tau cleavage at D421, tau detachment from microtubules, compromised axonal transport, and dysregulation of neuronal excitability [32, 34]. Moreover, transgenic mice expressing V337M human tau exhibited neurodegeneration and p-tau accumulation in the hippocampus, as well as behavioral abnormalities [54]. However, little was known about cleaved tau in the iN model besides the D421 cleavage site. The mAb.D402 and mAb.D13 neoepitope antibodies facilitated the detection of tau species cleaved by caspase-6, a protease closely associated with NFTs and neuronal loss in the human AD brain, including CA1 of the hippocampus, entorhinal cortex, the olfactory bulb, and brainstem $[15,23,28,29]$. Our results highlight and support previous suggestions of a central role of active caspase- 6 in tauopathies.

Several of the differences detected between tau ${ }^{\mathrm{V} 337 \mathrm{M}}$ and tau ${ }^{\mathrm{WT}}$ iNs only became apparent in iNs cultured for at least two or even three months. Western blot protein analysis of 1-3-month cultured iNs revealed a time-dependent upregulation of T18 oligomeric tau and MC1 conformational tau at two months in the $\operatorname{tau}^{\mathrm{V} 337 \mathrm{M}}$ iNs relative to controls. P-tau levels recognized by the PHF-1 antibody increased in the tau ${ }^{\mathrm{V} 337 \mathrm{M}}$ iNs relative to controls at three months, but not earlier. Physiological upregulation of $p$-tau in developing neurons is a known phenomenon in vitro and in human brains. Here, high levels of p-tau in tau ${ }^{W T}$ iNs only started to subside at two months post-differentiation. Future studies using iNs would benefit from using more mature neurons, despite the technical difficulties in maintaining neuronal lines in culture long-term.

Some of our results were intriguing and unexpected. Despite observing similar morphological and biochemical features in our tau ${ }^{\mathrm{V} 337 \mathrm{M}}$ iNs compared to other studies using tau ${ }^{\mathrm{V} 337 \mathrm{M}}$ lines, not all previously described changes were reproduced in our cells. We did not detect tau ${ }^{\mathrm{V} 337 \mathrm{M}}$-specific neurite length changes in early-differentiated iNs, as reported in another tau ${ }^{\mathrm{V} 337 \mathrm{M}}$ model [34]. This is likely due to different neuronal induction methodologies and resulting cell phenotypes. Although our results strongly suggest a significant role for caspase- 6 relative to other effector caspases in tau truncation and neurotoxicity, we could not demonstrate with certainty which caspase(s) were responsible for tau cleavage in our cell model because the peptidic z-VEID-fmk inhibitor is only partially selective for caspase-6 [48] and z-VAD-fmk caspase inhibitors are nonselective. Our understanding of the mechanisms of action of caspase inhibitors would tremendously benefit from follow up studies using highly selective, nonpeptidic inhibitors of caspase -6 that are currently under development. Moreover, exploration of apoptosis-related substrates targeted by caspases -3 and -6 in addition to tau, including nuclear and 
mitochondrial proteins, could further clarify the mechanisms of caspase-mediated neurotoxicity in our cell model.

In summary, our study demonstrates a time-dependent increase of tau cleavage and a pronounced vulnerability to stress and neuronal death in the tau ${ }^{\mathrm{V} 337 \mathrm{M}}$ iNs that is pharmacologically reversed by caspase inhibition. Our data support a model in which tau ${ }^{\mathrm{V} 337 \mathrm{M}}$ leads to activation of caspases, which in turn leads to increased vulnerability to toxic insult, including cleavage of tau to toxic or aggregation-prone tau species. While both tau ${ }^{\mathrm{V} 337 \mathrm{M}}$ and tau ${ }^{\mathrm{WT}}$ iNs respond to STS by activating caspases, tau ${ }^{\mathrm{V} 337 \mathrm{M}} \mathrm{iNs}$ are more vulnerable to tau proteolysis and cell death after caspase activation. Future mechanistic studies of caspase-dependent pathways across MAPT genotypes in iN models could reveal viable therapeutic targets against tau pathogenesis in FTLD and other tauopathies.

\section{Conclusions}

The mechanisms leading to tau pathology in frontotemporal dementia (FTD) and Alzheimer's disease (AD) remain elusive. Our data demonstrates that iN cultured for three months better model tau pathology than shorter culture times. Results with three-month old cultures of iNs bearing the V337M MAPT mutation demonstrate the deleterious role of caspase activation in tau pathology, the activation of caspase- 6 in particular, and the therapeutic potential of caspase inhibitors against tau-related neurodegenerative pathology.

\section{Declarations}

\section{Ethics approval and consent to participate}

Use of human brain tissue and iPSC-derived induced neurons from humans have been approved by the University of California San Francisco Institutional Review Board.

\section{Consent for publication}

Not applicable, the manuscript does not contain data from any individual person.

\section{Availability of data and materials}

The data that support the findings of this study are available from the corresponding authors upon reasonable request.

\section{Competing interests}

Michelle R. Arkin is cofounder of Elgia Therapeutics, which is developing caspase- 6 inhibitors for inflammatory diseases. The other authors have declared no conflict of interest.

\section{Funding}


This study was supported by the National Institutes of Health K01AG053433 (P.T), K24AG053435, R56AG057528 and U54 NS100717 (L.T.G), P30AG062422 and P01AG019724 (B.L.M), R01AG054025 (R.K), UCSF RAP Pilot Award program (P.T), UCSF RAP Team Science Grant (L.T.G., M.R.A.), Alzheimer's Association AARG-16-441514 (L.T.G., M.R.A.), Rainwater Charitable Foundation (J.E.G; M.R.A.), and a Catalyst award from ShangPharma Innovation (M.R.A., T.Y., S.K, R.N.).

\section{Authors' contributions}

P.T., M.R.A., and L.T.G. designed the study; P.T. performed the experiments, data acquisition and analyses. B.C., T.Y., S. K., R.N., A.J.A., and M.R.A. generated the neoepitope caspase-cleaved tau antibodies. R.K. provided the oligomeric tau antibody and contributed to data interpretation. C.W. and L.G. provided the iPSC lines, C.P. contributed to data analyses and graphic design, D.B., C.W., D.O.M., C.M.K, J.E.G., and S.T. contributed with cell culture work and data interpretation, B.L.M and L.T.G provided the human brain specimens, P.T. wrote the manuscript, C.W., D.B., R.N., S.T., M.R.A., and L.T.G. revised the manuscript, L.T.G. and M.R.A. supervised the study and approved the submitted version. All authors read and approved the final manuscript.

\section{Acknowledgments}

We thank Wing Hung Lee, Mifrah Hayath, Grace Pohan, Steven Chen, and Daniel Medina-Cleghorn for technical support; Dr. Andrea LeBlanc for helpful discussions on caspase biochemistry; Dr. Peter Davies for generously providing tau antibodies.

\section{Abbreviations}

AD: Alzheimer's disease

Asp: aspartic acid

ELISA: enzyme-linked immunosorbent assay

FTLD: frontotemporal lobar degeneration

HAT: hypoxanthine-aminopterin-thymidine

ICC: immunocytochemistry

IF: immunofluorescence

iNs: induced cortical neurons

iPSCs: induced pluripotent stem cells

KLH: keyhole limpet hemocyanin 
LDH: lactate dehydrogenase

mAbs: monoclonal antibodies

MAP2: microtubule-associated protein 2

MAPT: Microtubule-associated protein tau

NFT: neurofibrillary tangle

Ngn2: Neurogenin 2

p-tau: tau hyperphosphorylation

PHFs: paired helical filaments

PTMs: post-translational modifications

STS: staurosporine

tau ${ }^{\text {V337M: }}$ V337M MAPT mutation

tau $^{\text {WT. WT } M A P T}$

VGlut1: vesicular glutamate transporter 1

\section{References}

1. Lanata SC, Miller BL. The behavioural variant frontotemporal dementia (bvFTD) syndrome in psychiatry. J Neurol Neurosurg Psychiatry. 2016;87:501-11.

2. Spillantini MG, Goedert M. Tau pathology and neurodegeneration. Lancet Neurol. 2013;12:609-22.

3. Alonso AC, Grundke-Iqbal I, Iqbal K. Alzheimer's disease hyperphosphorylated tau sequesters normal tau into tangles of filaments and disassembles microtubules. Nat Med. 1996;2:783-7.

4. Iqbal K, Grundke-Iqbal I, Zaidi T, Merz PA, Wen GY, Shaikh SS, et al. Defective brain microtubule assembly in Alzheimer's disease. Lancet Lond Engl. 1986;2:421-6.

5. de Calignon A, Fox LM, Pitstick R, Carlson GA, Bacskai BJ, Spires-Jones TL, et al. Caspase activation precedes and leads to tangles. Nature. 2010;464:1201-4.

6. Kim Y, Choi H, Lee W, Park H, Kam T-I, Hong S, et al. Caspase-cleaved tau exhibits rapid memory impairment associated with tau oligomers in a transgenic mouse model. Neurobiol Dis. 2016;87:1928.

7. Nicholls SB, DeVos SL, Commins C, Nobuhara C, Bennett RE, Corjuc DL, et al. Characterization of TauC3 antibody and demonstration of its potential to block tau propagation. PLOS ONE Public Library of Science. 2017;12:e0177914. 
8. Rissman RA, Poon WW, Blurton-Jones M, Oddo S, Torp R, Vitek MP, et al. Caspase-cleavage of tau is an early event in Alzheimer disease tangle pathology. J Clin Invest. 2004;114:121-30.

9. Enari M, Sakahira H, Yokoyama H, Okawa K, Iwamatsu A, Nagata S. A caspase-activated DNase that degrades DNA during apoptosis, and its inhibitor ICAD. Nature Nature Publishing Group. 1998;391:43-50.

10. Lakhani SA, Masud A, Kuida K, Porter GA, Booth CJ, Mehal WZ, et al. Caspases 3 and 7: Key Mediators of Mitochondrial Events of Apoptosis. 311: Science. American Association for the Advancement of Science; 2006. pp. 847-51.

11. Thornberry NA, Lazebnik Y. Caspases: enemies within. Science. 1998;281:1312-6.

12. Albrecht S, Bourdeau M, Bennett D, Mufson EJ, Bhattacharjee M, LeBlanc AC. Activation of caspase-6 in aging and mild cognitive impairment. Am J Pathol. 2007;170:1200-9.

13. Girling KD, Demers M-J, Laine J, Zhang S, Wang YT, Graham RK. Activation of caspase- 6 and cleavage of caspase- 6 substrates is an early event in NMDA receptor-mediated excitotoxicity. $J$ Neurosci Res. 2018;96:391-406.

14. Graham RK, Deng Y, Slow EJ, Haigh B, Bissada N, Lu G, et al. Cleavage at the caspase- 6 site is required for neuronal dysfunction and degeneration due to mutant huntingtin. Cell. 2006;125:117991.

15. Theofilas P, Ehrenberg AJ, Nguy A, Thackrey JM, Dunlop S, Mejia MB, et al. Probing the correlation of neuronal loss, neurofibrillary tangles, and cell death markers across the Alzheimer's disease Braak stages: a quantitative study in humans. Neurobiol Aging. 2018;61:1-12.

16. Ladha S, Qiu X, Casal L, Caron NS, Ehrnhoefer DE, Hayden MR. Constitutive ablation of caspase- 6 reduces the inflammatory response and behavioural changes caused by peripheral pro-inflammatory stimuli. Cell Death Discov Nature Publishing Group. 2018;4:1-10.

17. Gamblin TC, Chen F, Zambrano A, Abraha A, Lagalwar S, Guillozet AL, et al. Caspase cleavage of tau: linking amyloid and neurofibrillary tangles in Alzheimer's disease. Proc Natl Acad Sci U S A. 2003;100:10032-7.

18. Horowitz PM, Patterson KR, Guillozet-Bongaarts AL, Reynolds MR, Carroll CA, Weintraub ST, et al. Early N-terminal changes and caspase- 6 cleavage of tau in Alzheimer's disease. J Neurosci Off J Soc Neurosci. 2004;24:7895-902.

19. Zhang Q, Zhang X, Sun A. Truncated tau at D421 is associated with neurodegeneration and tangle formation in the brain of Alzheimer transgenic models. Acta Neuropathol (Berl). 2009;117:687-97.

20. Zhao Y, Tseng I-C, Heyser CJ, Rockenstein E, Mante M, Adame A, et al. Appoptosin-Mediated Caspase Cleavage of Tau Contributes to Progressive Supranuclear Palsy Pathogenesis. Neuron. 2015;87:963-75.

21. Cusack CL, Swahari V, Hampton Henley W, Michael Ramsey J, Deshmukh M. Distinct pathways mediate axon degeneration during apoptosis and axon-specific pruning. Nat Commun. 2013;4:1876.

22. Nikolaev A, McLaughlin T, O'Leary DDM, Tessier-Lavigne M. APP binds DR6 to trigger axon pruning and neuron death via distinct caspases. Nature. 2009;457:981-9. 
23. Ramcharitar J, Albrecht S, Afonso VM, Kaushal V, Bennett DA, Leblanc AC. Cerebrospinal fluid tau cleaved by caspase- 6 reflects brain levels and cognition in aging and Alzheimer disease. J Neuropathol Exp Neurol. 2013;72:824-32.

24. Angel A, Volkman R, Royal TG, Offen D. Caspase-6 Knockout in the 5xFAD Model of Alzheimer's Disease Reveals Favorable Outcome on Memory and Neurological Hallmarks. Int J Mol Sci. 2020;21.

25. Klaiman G, Petzke TL, Hammond J, Leblanc AC. Targets of caspase-6 activity in human neurons and Alzheimer disease. Mol Cell Proteomics. 2008;7:1541-55.

26. Cotman CW, Poon WW, Rissman RA, Blurton-Jones M. The role of caspase cleavage of tau in Alzheimer disease neuropathology. J Neuropathol Exp Neurol. 2005;64:104-12.

27. Novak M, Kabat J, Wischik CM. Molecular characterization of the minimal protease resistant tau unit of the Alzheimer's disease paired helical filament. EMBO J. 1993;12:365-70.

28. Foveau B, Albrecht S, Bennett DA, Correa JA, LeBlanc AC. Increased Caspase-6 activity in the human anterior olfactory nuclei of the olfactory bulb is associated with cognitive impairment. Acta Neuropathol Commun. 2016;4:127.

29. Guo H, Albrecht S, Bourdeau M, Petzke T, Bergeron C, LeBlanc AC. Active caspase-6 and caspase-6cleaved tau in neuropil threads, neuritic plaques, and neurofibrillary tangles of Alzheimer's disease. Am J Pathol. 2004;165:523-31.

30. Hong M, Zhukareva V, Vogelsberg-Ragaglia V, Wszolek Z, Reed L, Miller BI, et al. Mutation-specific functional impairments in distinct tau isoforms of hereditary FTDP-17. Science. 1998;282:1914-7.

31. Karch CM, Kao AW, Karydas A, Onanuga K, Martinez R, Argouarch A, et al. A Comprehensive Resource for Induced Pluripotent Stem Cells from Patients with Primary Tauopathies. Stem Cell Rep. 2019;13:939-55.

32. Sohn PD, Huang CT-L, Yan R, Fan L, Tracy TE, Camargo CM, et al. Pathogenic tau impairs axon initial segment plasticity and excitability homeostasis. Neuron. 2019;104:458-70.e5.

33. Spina S, Schonhaut DR, Boeve BF, Seeley WW, Ossenkoppele R, O'Neil JP, et al. Frontotemporal dementia with the V337M MAPT mutation: Tau-PET and pathology correlations. Neurology. 2017;88:758-66.

34. Ehrlich M, Hallmann A-L, Reinhardt P, Araúzo-Bravo MJ, Korr S, Röpke A, et al. Distinct neurodegenerative changes in an induced pluripotent stem cell model of frontotemporal dementia linked to mutant TAU protein. Stem Cell Rep. 2015;5:83-96.

35. Jiang S, Wen N, Li Z, Dube U, Del Aguila J, Budde J, et al. Integrative system biology analyses of CRISPR-edited iPSC-derived neurons and human brains reveal deficiencies of presynaptic signaling in FTLD and PSP. Transl Psychiatry. 2018;8:265.

36. Nakamura M, Shiozawa S, Tsuboi D, Amano M, Watanabe H, Maeda S, et al. Pathological Progression Induced by the Frontotemporal Dementia-Associated R406W Tau Mutation in PatientDerived iPSCs. Stem Cell Rep [Internet]. 2019 [cited 2019 Oct 14]; Available from: http://dx.doi.org/10.1016/j.stemcr.2019.08.011. 
37. Silva MC, Cheng C, Mair W, Almeida S, Fong H, Biswas MHU, et al. Human iPSC-Derived Neuronal Model of Tau-A152T Frontotemporal Dementia Reveals Tau-Mediated Mechanisms of Neuronal Vulnerability. Stem Cell Rep. 2016;7:325-40.

38. Wang C, Ward ME, Chen R, Liu K, Tracy TE, Chen X, et al. Scalable Production of iPSC-Derived Human Neurons to Identify Tau-Lowering Compounds by High-Content Screening. Stem Cell Rep. 2017;9:1221-33.

39. Miyaoka Y, Chan AH, Judge LM, Yoo J, Huang M, Nguyen TD, et al. Isolation of single-base genomeedited human iPS cells without antibiotic selection. Nat Methods. 2014;11:291-3.

40. Ehrnhoefer DE, Skotte NH, Savill J, Nguyen YTN, Ladha S, Cao L-P, et al. A Quantitative Method for the Specific Assessment of Caspase-6 Activity in Cell Culture. PLOS ONE Public Library of Science. 2011;6:e27680.

41. Zhang Y, Pak C, Han Y, Ahlenius H, Zhang Z, Chanda S, et al. Rapid single-step induction of functional neurons from human pluripotent stem cells. Neuron. 2013;78:785-98.

42. Hefti MM, Farrell K, Kim S, Bowles KR, Fowkes ME, Raj T, et al. High-resolution temporal and regional mapping of MAPT expression and splicing in human brain development. PLOS ONE Public Library of Science. 2018;13:e0195771.

43. Iovino M, Agathou S, González-Rueda A, Del Castillo Velasco-Herrera M, Borroni B, Alberici A, et al. Early maturation and distinct tau pathology in induced pluripotent stem cell-derived neurons from patients with MAPT mutations. Brain J Neurol. 2015;138:3345-59.

44. Puangmalai N, Bhatt N, Montalbano M, Sengupta U, Gaikwad S, Ventura F, et al. Internalization mechanisms of brain-derived tau oligomers from patients with Alzheimer's disease, progressive supranuclear palsy and dementia with Lewy bodies. Cell Death Dis. 2020;11:314.

45. Lo Cascio F, Garcia S, Montalbano M, Puangmalai N, McAllen S, Pace A, et al. Modulating DiseaseRelevant Tau Oligomeric Strains by Small Molecules. J Biol Chem. 2020.

46. Jicha GA, Bowser R, Kazam IG, Davies P. Alz-50 and MC-1, a new monoclonal antibody raised to paired helical filaments, recognize conformational epitopes on recombinant tau. J Neurosci Res. 1997;48:128-32.

47. Mondragón-Rodríguez S, Perry G, Luna-Muñoz J, Acevedo-Aquino MC, Williams S. Phosphorylation of tau protein at sites Ser(396-404) is one of the earliest events in Alzheimer's disease and Down syndrome. Neuropathol Appl Neurobiol. 2014;40:121-35.

48. Mintzer R, Ramaswamy S, Shah K, Hannoush RN, Pozniak CD, Cohen F, et al. A whole cell assay to measure caspase- 6 activity by detecting cleavage of lamin A/C. Plos One. 2012;7:e30376.

49. Wheeler HE, Wing C, Delaney SM, Komatsu M, Dolan ME. Modeling Chemotherapeutic Neurotoxicity with Human Induced Pluripotent Stem Cell-Derived Neuronal Cells. PLOS ONE Public Library of Science. 2015;10:e0118020.

50. Guillozet-Bongaarts AL, Cahill ME, Cryns VL, Reynolds MR, Berry RW, Binder LI.

Pseudophosphorylation of tau at serine 422 inhibits caspase cleavage: in vitro evidence and implications for tangle formation in vivo. J Neurochem. 2006;97:1005-14. 
51. Fiock KL, Smalley ME, Crary JF, Pasca AM, Hefti MM. Increased Tau Expression Correlates with Neuronal Maturation in the Developing Human Cerebral Cortex. eNeuro [Internet]. Society for Neuroscience; 2020 [cited 2020 Aug 13];7. Available from: https://www.eneuro.org/content/7/3/ENEURO.0058-20.2020.

52. Zhang Y, Goodyer C, LeBlanc A. Selective and protracted apoptosis in human primary neurons microinjected with active caspase- $3,-6,-7$, and -8 . J Neurosci Off J Soc Neurosci UNITED STATES. 2000;20:8384-9.

53. LeBlanc A, Liu H, Goodyer C, Bergeron C, Hammond J. Caspase-6 role in apoptosis of human neurons, amyloidogenesis, and Alzheimer's disease. J Biol Chem. 1999;274:23426-36.

54. Tanemura K, Murayama M, Akagi T, Hashikawa T, Tominaga T, Ichikawa M, et al. Neurodegeneration with Tau Accumulation in a Transgenic Mouse Expressing V337M Human Tau. J Neurosci. 2002;22:133-41.

\section{Figures}


a

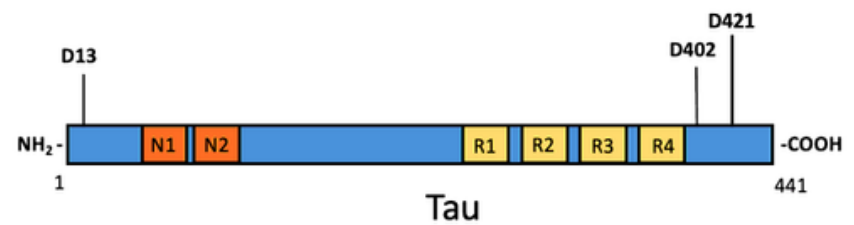

b

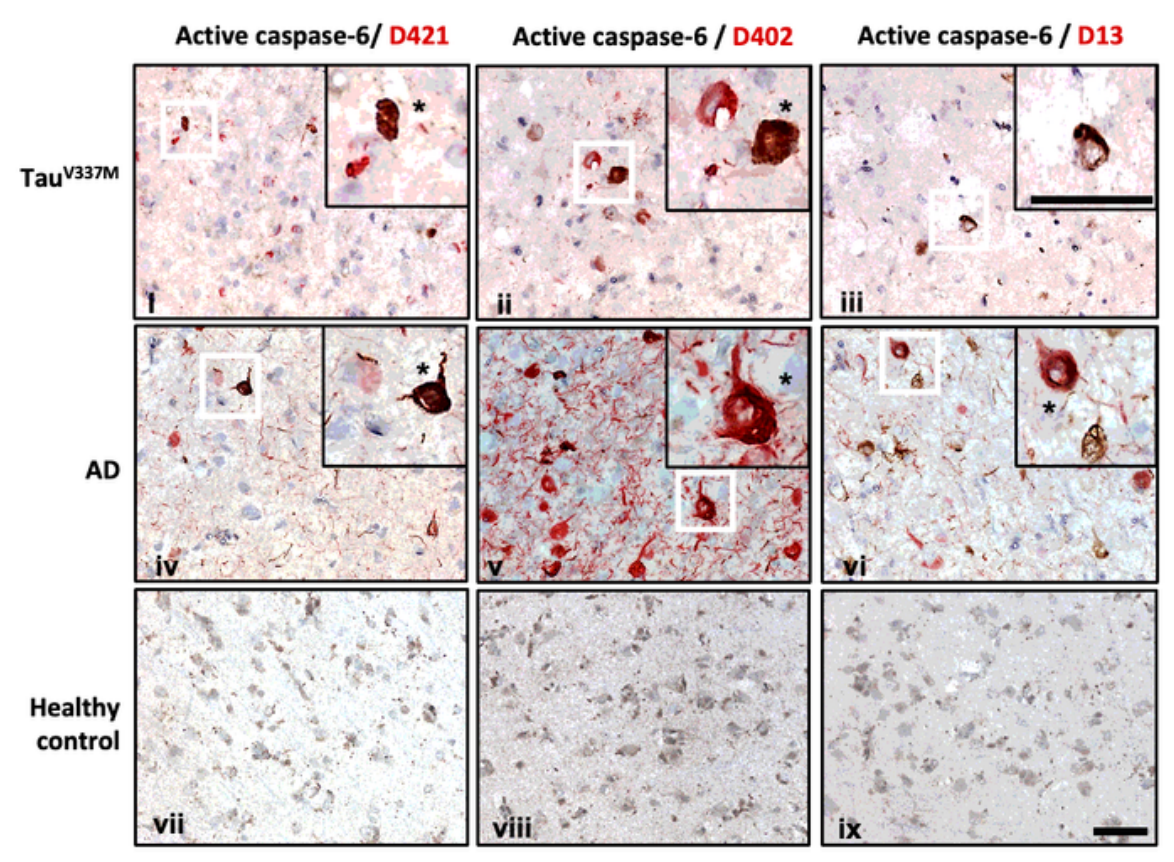

Fig. 1

Figure 1

Caspase- 6 cleaved tau neoepitope antibody positivity in human postmortem brains with tau accumulation. (a) Schematic depicting two caspase- 6 cleaved tau sites targeted by monoclonal neoepitope antibodies mAb.D13 (D13; 14-441) and mAb.D402 (D402; 1-402), at the $\mathrm{N}$ - and C-terminus of tau, respectively. The C-terminus D421 site of tau is targeted by the antibody TauC3 and cleaved by multiple caspases; (b) Brain sections from the temporal cortex of an individual with tauV337M and FTD 
(i-iii), and a patient with sporadic late-stage AD (iv-vi) showing antibody positivity for active caspase-6 (brown) and caspase-cleaved tau (red), including TauC3 (D421) (i, iv), mAb.D402 (ii, v) and mAb.D13 (iii, vi). No antibody positivity was observed in an elderly healthy control (vii-ix), even after prolonged incubation with the chromogen to ensure the absence of antibody signal. Insets represent the magnification of representative neurons (white squares). Asterisks indicate cells positive for both active caspase- 6 and caspase-cleaved tau markers. Scale bars: $50 \mu \mathrm{m}$

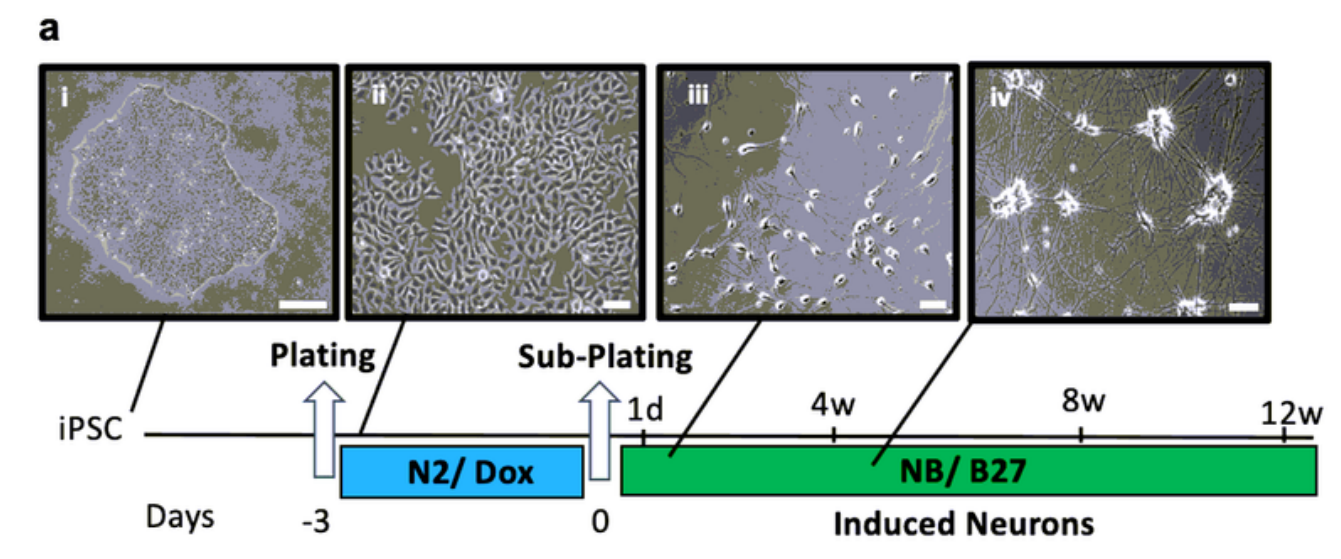

b

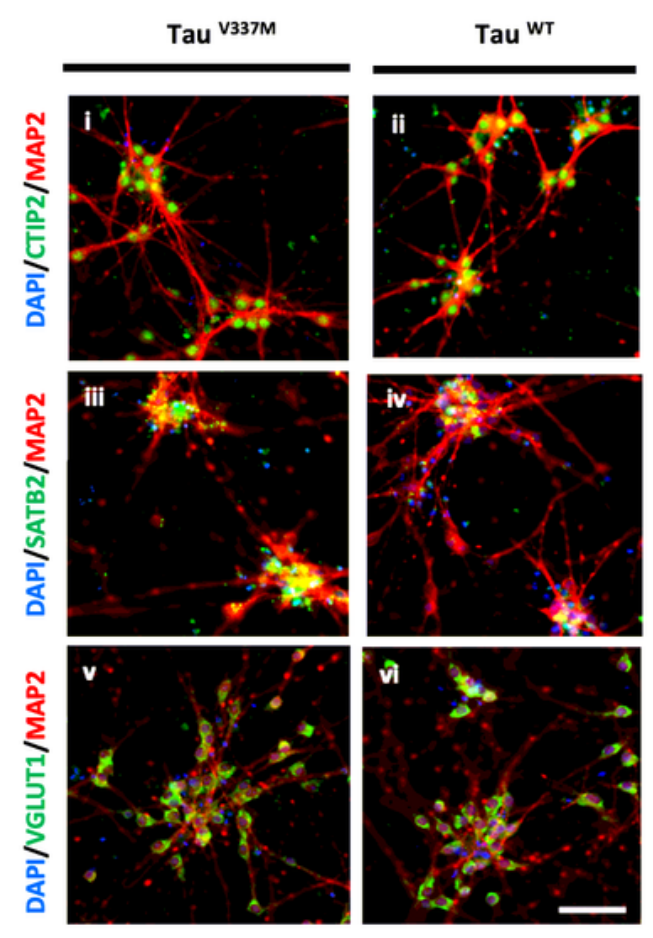

Fig. 2

Figure 2 
Timeline of iPSC differentiation to neurons following doxycycline-inducible expression of Ngn2. (a) Phase-contrast images represent individual stages of the differentiation protocol, including iPSCs (i), neuronal precursors (ii), immature neurons (iii), and mature neurons (iv); (b) IF characterization of neurons at 1-month post differentiation. Mutant (tauV337M) and control (tauWT) neurons were positive for cortical (green; i-iv), glutamatergic (green; v-vi), and neuronal (red; i-vi) markers. Nuclei were stained with DAPI (blue; i-vi). Scale bars: (a) i: $200 \mu \mathrm{m}$, ii-iv: $50 \mu \mathrm{m}$ (b) $60 \mu \mathrm{m}$.
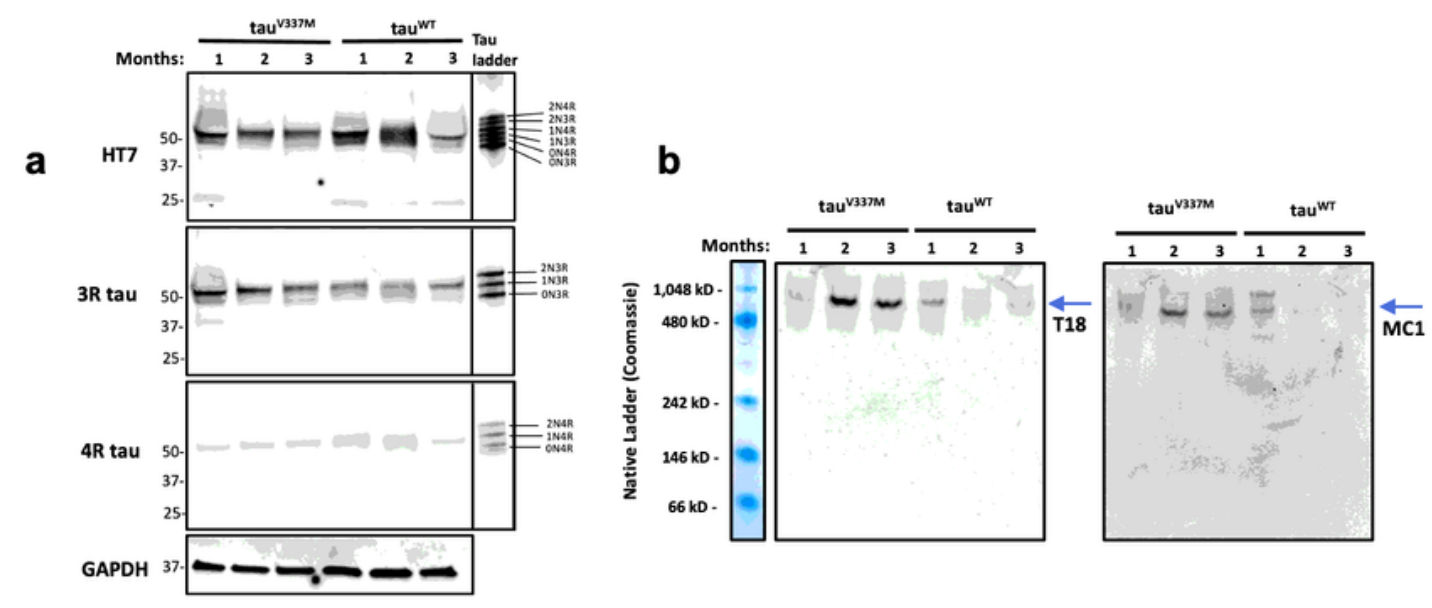

Fig. 3
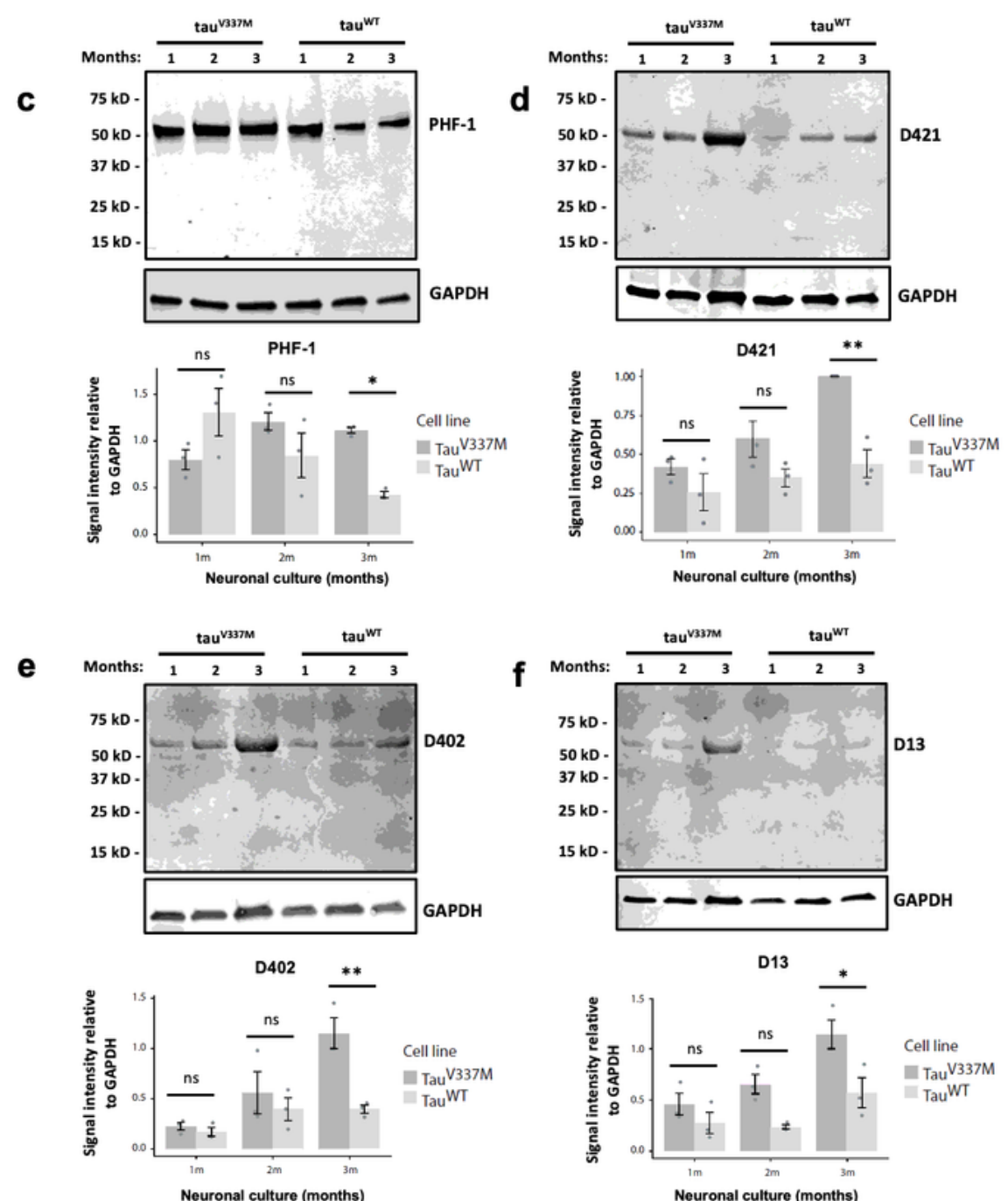

Figure 3 
Time-dependent tau pathological changes in the tauV337M neurons detected by western blot analyses. (a) Expression of total tau (HT7) and tau isoforms (3R and 4R tau) in tauV337M and tauWT neurons cultured from 1 to 3 months. Antibody specificity was confirmed by a recombinant tau ladder containing all six tau isoforms (right panels); (b) Oligomeric (T18) and conformational (MC1) tau levels were detected under non-denaturing conditions for preserving protein conformation. The molecular weight of the proteins was estimated using a protein standard for native electrophoresis stained with Coomassie blue (far-left panel) for band visualization (see also Fig. S4); (c-f) Semi-quantification of p-tau (PHF-1) and caspase-cleave tau (D421, D402, and D13) protein levels based on band intensities relative to GAPDH internal loading control ( $\mathrm{n} \geq 3$ independent experiments; two-way ANOVA with post hoc Tukey test; ns, not significant: $\left.p>0.05 ;{ }^{*} p<0.05,{ }^{* *} p<0.01\right)$. 


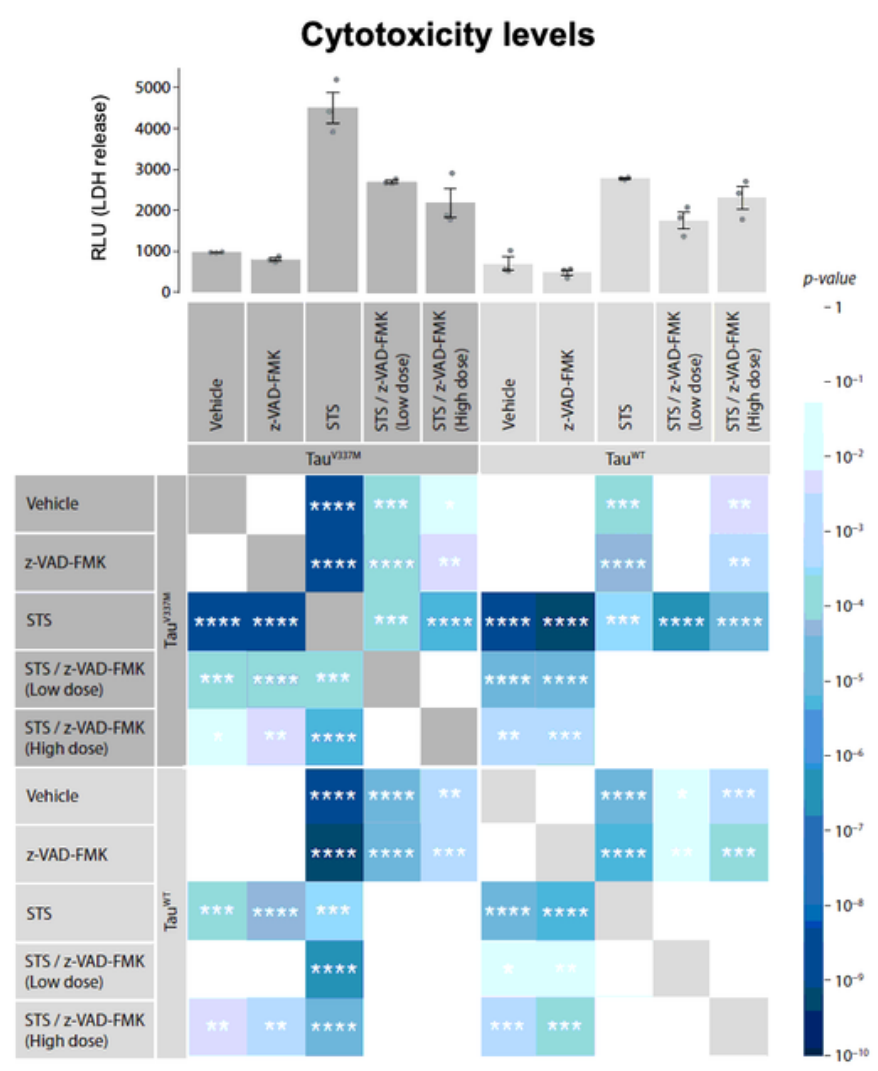

Fig. 4

Figure 4

Caspase inhibition is neuroprotective against acute stress-induced cytotoxicity in the tauV337M neurons. Changes in cytotoxicity levels were measured by LDH release following a $48 \mathrm{~h}$ treatment with STS and the pan-caspase inhibitor (z-VAD-fmk) in 3-month cultured neurons. Matrix heatmap illustrates p-values (color gradient) and significance levels (asterisks) between treatment groups. Dark gray shade represents 
tauV337M and light gray shade represents tauWT neurons ( $\mathrm{n}=3$ independent experiments; two-way ANOVA with post hoc Tukey test; $\left.{ }^{*} p<0.05,{ }^{*} p<<0.01,{ }^{\star \star *} p<0.001,{ }^{* \star \star *} p<0.0001\right)$. RLU: Relative light units.
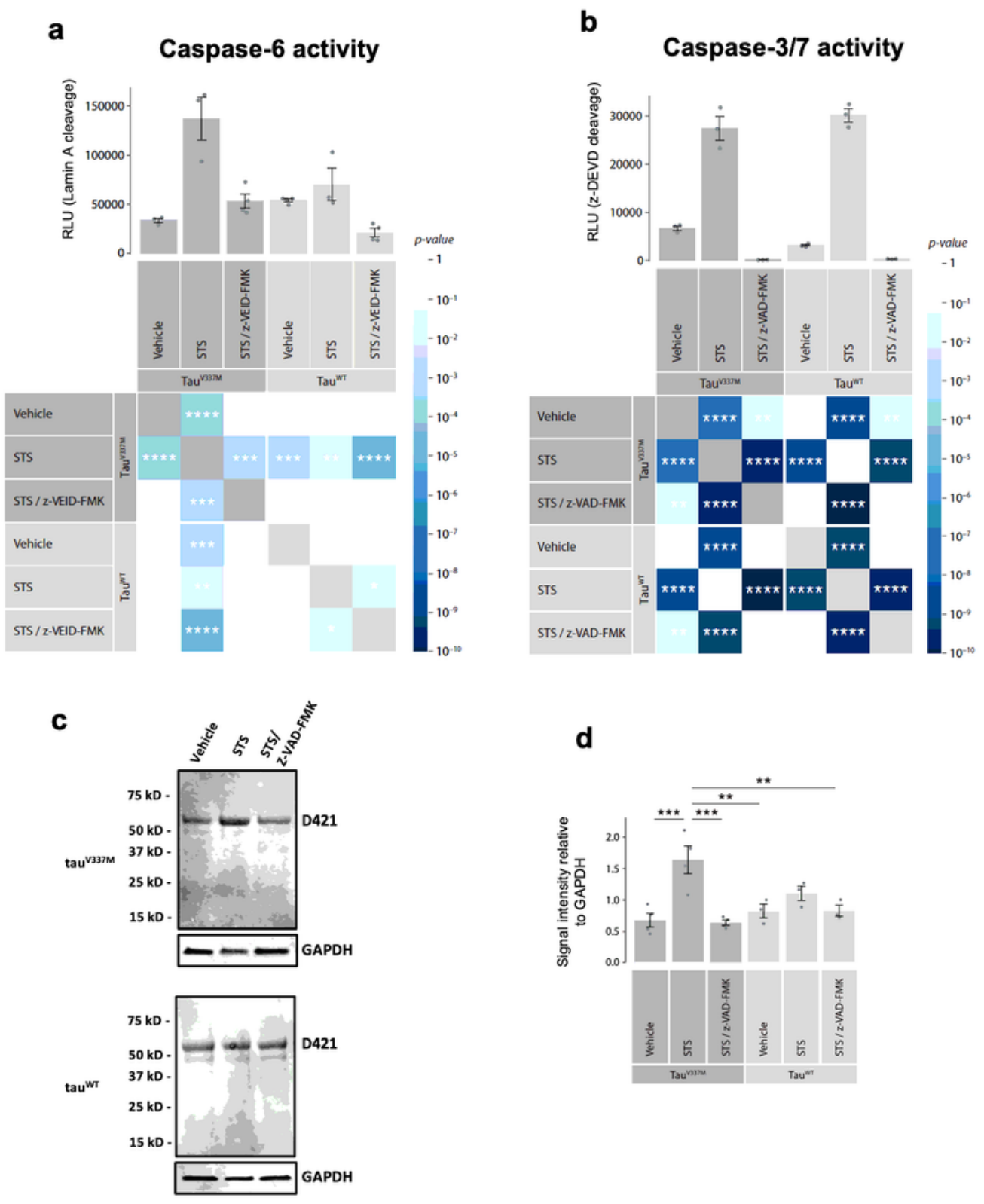

\section{Fig. 5}

\section{Figure 5}

Stress-induced caspase activation in the induced neurons. Active caspase-6 (a) and caspase-3/7 levels (b) were examined in 3-month cultured neurons using caspase-specific cleavage substrates following treatment with vehicle, STS, and STS/z-VEID-fmk (a caspase-6 inhibitor) or STS/z-VAD-fmk (a pan- 
caspase inhibitor). Matrix heatmap illustrates $p$-values (color gradient) and significance levels (asterisks) between treatment groups. Dark gray shade represents tauV337M and light gray shade represents tauWT neurons; (c-d) Semi-quantification of caspase-cleaved tau (D421) protein levels in neurons treated with STS or STS/z-VAD-fmk for $48 \mathrm{~h}$ based on band intensities relative to GAPDH internal loading control $(\mathrm{n} \geq$ 3 independent experiments; two-way ANOVA with post hoc Tukey test; ${ }^{\star} p<0.05,{ }^{*} p<0.01,{ }^{\star \star \star} p<0.001$, $\star \star \star \star p<0.0001)$. RLU: Relative light units.

a
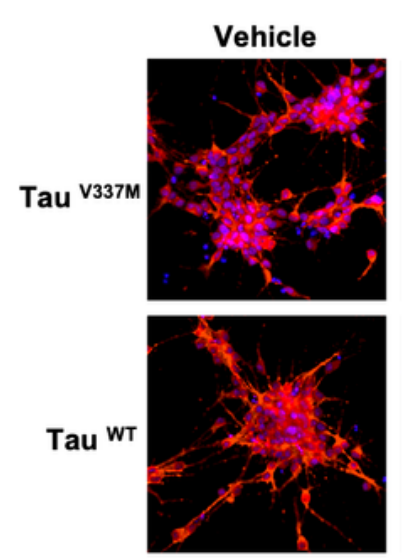

STS
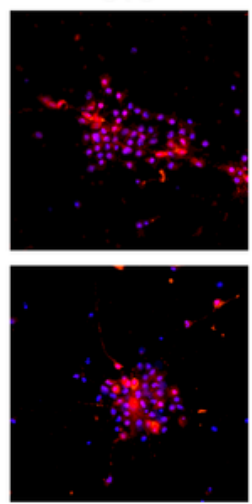

STS/ Z-VAD-FMK
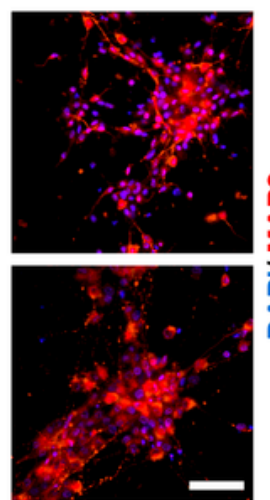

b

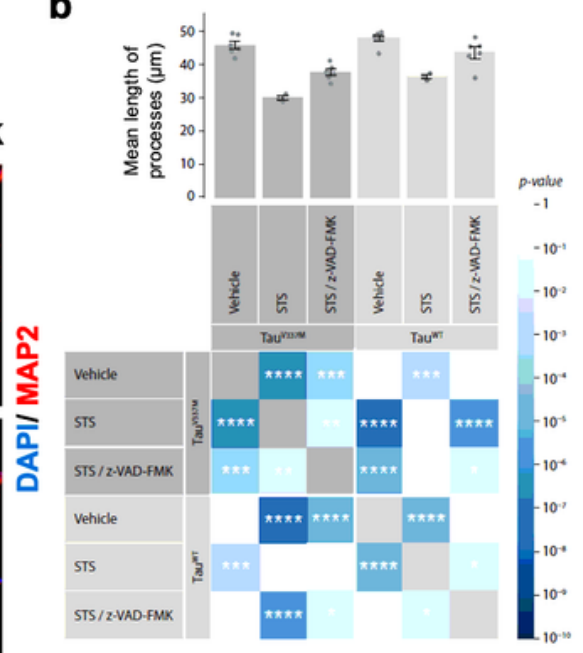

Fig. 6

Figure 6 
Caspase inhibition rescues stress-induced reduction of neurite length in the induced neurons. (a) IF staining of neurites (MAP2, red) and cell nuclei (DAPI, blue) following a 48h treatment with STS and caspase-inhibitor (z-VAD-fmk) in 3-month cultured neurons; (b) Image quantification of mean length of processes (based on a). Matrix heatmap illustrates p-values (color gradient) and significance levels (asterisks) between treatment groups. Dark gray shade represents tauV337M and light gray shade represents tauWT neurons ( $\mathrm{n} \geq 3$ independent experiments; two-way ANOVA with post hoc Tukey test; * $p$ $\left.<0.05,{ }^{*} p<0.01,{ }^{\star \star *} p<0.001,{ }^{* \star \star *} p<0.0001\right)$. Scale bar: $50 \mu \mathrm{m}$.

\section{Supplementary Files}

This is a list of supplementary files associated with this preprint. Click to download.

- SupplementalExperimentalProcedures.pdf

- Figures1.pdf

- Figures2.pdf

- Figures3.pdf

- FigureS4.pdf

- FigureS5.pdf

- FigureS6.pdf 